\title{
Kayseri ili merkez ilçelerinde süs bitkileri üzerinde bulunan yaprakbitleri (Hemiptera: Aphididae)'nin parazitoitleri ve avcı Coccinellidleri ${ }^{1}$
}

\author{
Deniz Özkan ÖZTÜRK², Murat MUŞTU²
}

Parasitoids and predaceous coccinellids of aphids (Hemiptera: Aphididae) on ornamental plants in central districts of Kayseri Province

\begin{abstract}
The present study was conducted to determine the parasitoids and predaceous coccinellids of aphids on trees and shrubs from parks, roadside plantings and landscaped areas in the central districts (Melikgazi, Kocasinan, Talas, Hacilar, Incesu) of Kayseri Province between the years 2014 and 2015.

Nine parasitoid species were collected and one more species was identified to the genus level. They are Aphidius sp., A. hortensis Marshall 1896, A. matricariae Haliday 1834, A. urticae Haliday 1834, A. rosae Haliday 1834, Binodoxys angelicae Haliday 1833, Lysiphlebus fabarum Marshall 1896, L. testaceipes Cresson 1880, Praon abjectum Haliday 1833 and $P$. volucre Haliday 1833. In addition, 11 species of aphid predators from the Coccinellidae were collected. They are Adalia (Adalia) bipunctata (L.) 1758, A. (Adalia) decempunctata (L.) 1758, A. fasciatopunctata revelierei (Mulsant) 1866, Chilocorus bipustulatus (L.) 1758 , Coccinella (Coccinella) septempunctata L. 1758, Exochomus (Exochomus) quadripustulatus (L.) 1758, Hippodamia (Adonia) variegata (Goeze) 1777, Oenopia (Synharmonia) conglobata (L.) 1758, Propylea quatuordecimpunctata (L.) 1758, Scymnus (Scymnus) apetzi Mulsant 1846 and S. (Pullus) subvillosus (Goeze) 1777.
\end{abstract}

Key words: Natural enemy, fauna, survey, ornamental plant, aphid

Özet: Bu çalışma, 2014 ve 2015 yılları arasında Kayseri ili merkez ilçelerindeki (Melikgazi, Kocasinan, Talas, Hacılar, İncesu) park, yol plantasyonu ve peyzaj alanlarındaki ağaç ve çalılar üzerindeki yaprakbitlerinin parazitoit ve avcı Coccinellidlerini belirlemek için yürütülmüştür.

$\mathrm{Bu}$ sörvey sonucunda, dokuz parazitoit tür tespit edilmiş, bir parazitoit ise cins düzeyinde teşhis edilebilmiştir. Bu türler Aphidius sp., A. hortensis Marshall 1896, A. matricariae Haliday 1834, A. urticae Haliday 1834, A. rosae Haliday 1834, Binodoxys angelicae Haliday 1833, Lysiphlebus fabarum Marshall 1896, L. testaceipes Cresson 1880, Praon abjectum Haliday 1833 and P. volucre Haliday 1833'dir. Ayrıca, yaprakbiti avcısı olarak Coccinellidae

\footnotetext{
${ }^{2}$ Erciyes Üniversitesi, Seyrani Ziraat Fakültesi, Bitki Koruma Bölümü - 38280, Talas, Kayseri Sorumlu yazar (Corresponding author) e-mail: mmustu77@ hotmail.com Alınış (Recieved): 21.02.2018 
Kayseri ili süs bitkilerindeki yaprakbitlerinin parazitoitleri ve avc1 Coccinellidleri

familyasından 11 tür belirlenmiştir. Bu türler Adalia (Adalia) bipunctata (L.) 1758, A. (Adalia) decempunctata (L.) 1758, A. fasciatopunctata revelierei (Mulsant) 1866, Chilocorus bipustulatus (L.) 1758, Coccinella (Coccinella) septempunctata L. 1758, Exochomus (Exochomus) quadripustulatus (L.) 1758, Hippodamia (Adonia) variegata (Goeze) 1777, Oenopia (Synharmonia) conglobata (L.) 1758, Propylea quatuordecimpunctata (L.) 1758, Scymnus (Scymnus) apetzi Mulsant 1846, S. (Pullus) subvillosus (Goeze) 1777'dır.

Anahtar sözcükler: Doğal düşman, fauna, sörvey, süs bitkisi, yaprakbiti

\section{Giriş}

Son yıllarda hızla artan kent nüfusu ve hızlı yapılaşma ile paralel olarak, kentlerde yaşayan insanların yaşam kalitesini arttırmak, kaybolan doğal hayatı yeniden oluşturmak ve görünüm güzelliği sağlamak için süs bitkilerinin kullanıldığ 1 park, bahçe ve yol kenarlarında oluşturulan yeşil alanların miktarı da her geçen gün artmaktadır. Bu alanlarda kullanılan başlıca dış mekân süs bitkileri ağaç ve çalı formundaki bitkilerdir. Bununla birlikte, birçoğu kullanıldığı bölge için yeni olan ya da doğal bulunma sıklığının çok üzerindeki sayılarda kullanılan bu bitkiler, çok sayıda zararlı ve hastalık etmeni tarafından saldırıya uğramaktadır. Bu zararlıların en önemli gruplarından birini de Hemiptera takımı içerisindeki Aphidoidea üst familyası içerisindeki yaprakbitleri oluşturmaktadır. Yaprakbitleri bu bitkilerin çeşitli aksamlarında bitki özsuyunu emmeleri, emgi sırasında bitkilere toksik maddeler enjekte etmeleri, bitkilerde şekil bozukluğu, gal ve ur gibi yapılara sebep olmaları fumajine neden olmaları ve bitki virüs hastalıklarının vektörü olmaları gibi nedenlerle bitkilerin gelişimini engellemekte, hatta kuruyup ölmelerine neden olabilmektedirler.

Farklı habitatlar içerisinde yayılım gösteren yaprakbitlerinin popülâsyonlarını sınırlayan çok sayıda doğal düşman bulunmaktadır. Bu doğal düşmanlar arasında yaprakbiti parazitoitleri ve avcı coccinellidler önemli iki grubu oluşturmaktadır. Yaprakbitleri, Hymenoptera takımının Braconidae ve Aphelinidae familyalarına ait birçok tür ve Diptera takımının Cecidomyiidae familyasına bağlı birkaç tür tarafindan parazitlenmekte olup, bütün Hymenoptera takımına bağlı yaprakbiti parazitoitleri soliter, koinobiont ve endoparazitoit türlerdir (Boivin et al. 2012). Günümüze kadar Endaphis (Diptera: Cecidomyiidae) cinsine bağlı 6 türü tanımlanmış olan Dipter yaprakbiti parazitoitleri de, koinobiont ve endoparazitoit türlerdir ve son larva döneminde konukçuyu terk edip toprakta pupa olurlar (Muratori et al. 2009). Ayrica Hymenopter parazitoitlerin aksine, Dipter parazitoitler ile parazitlenen yaprakbitlerinde mumya oluşumu görülmez (Boivin et al. 2012). Hymenopter yaprakbiti parazitoitlerinin büyük bir çoğunluğu Aphidiinae (Hymenoptera: Braconidae) alt familyasinda bulunmakta olup, bu alt familya bireyleri yaprakbitlerine özelleşmiş parazitoit türler olarak bilinmektedir (Boivin et al. 2012). Tüm dünyaya yayılmış olan Aphidiinae alt familyasında 50 cinse bağlı 400 tür bulunmaktadır (Smith \& Kambhampati 2000). 
Yaprakbitlerinin en önemli doğal düşman gruplarından biri de, Türkçede yaygın olarak uğurböceği ya da gelin böceği olarak adlandırılan Coccinellidae (Coleoptera) familyasına ait türlerdir. Coccinellidlerin yaklaşık \%90'1 larva ve ergin dönemlerinde diğer böcek ya da akar gibi canlılarla beslenen yararlı avc1lar iken, diğerleri bitkiler ya da funguslarla beslenirler (Iperti 1999). Coccinellidae familyası bireyleri ergin ve ergin öncesi dönemlerinde aynı beslenme alışkanlığına sahip olup, avc1 türlerin çoğu yaprakbitleri ve Coccoidlerle beslenmektedir. Dünyada hemen hemen tüm ekosistemlerde yaşayabilen Coccinellidae familyasına ait Afidofag türlerin çoğu ve tek döl veren türler göç etme eğilimindedir (Iperti 1999). Dünyada şu ana kadar 360 cinse bağlı 6000 Coccinellid türü tespit edilmiştir (Vandenberg 2002). Türkiye'de ise Coccinellidler ile ilgili yapılan çalışmalar hala sınırlı düzeyde olup, şu ana kadar 39 cinse bağlı 105 tür saptanmıştır (Oğuzoğlu et al. 2017). Bu çalışmada Kayseri ili merkez ilçelerinde park, bahçe ve yol kenarlarında bulunan ağaç ve çalı formundaki süs bitkilerinde zarar yapan yaprakbitlerinin parazitoitleri ve Coccinellidae familyasına ait avcılarının tespit edilmesi amaçlanmıştır.

\section{Materyal ve yöntem}

Örneklemeler 2014-2015 yılları Nisan-Ekim ayları arasında, periyodik olmayan arazi çıkışları şeklinde yapılmıştır. Örnekleme esnasında Kayseri ili merkez ilçelerinde park, yollar üzerindeki ağaçlar ve peyzaj alanlarında bulunan bitkilerin yaprak, dal ve gövdeleri üzerinde bulunan yaprakbiti kolonileri gözle kontrol edilmiş, koloniler üzerinde mumyalaşmış yaprakbitleri ve Coccinellid larva veya yumurtaları bulunan bitki parçaları kesilerek önce kese kağıdı ve sonra polietilen poşetler içerisine olmak üzere ayrı ayrı yerleştirilerek etiketlenmiş ve laboratuvara getirilmiştir. Ergin öncesi dönemde laboratuvara getirilen Coccinellidler beslenip ergin döneme ulaştırılmışlardır. Ergin dönemde yaprakbiti kolonileri üzerinde beslendiği görülen Coccinellidler emgi tüpü yardımı ile toplanmış ve etiketlenerek laboratuvara getirilmiştir.

\section{Parazitoitlerin belirlenmesi}

Üzerinde mumyalaşmış yaprakbitleri bulunan bitki parçaları laboratuvarda 3 lt'lik plastik kavanozların içinde ayrı ayrı olarak kültüre alınmış ve parazitoit çıkışları beklenmiştir. Plastik kavanozların üzeri bitkilerin hava almasını sağlamak amacıyla ince delikli tül ile örtülmüştür. Ergin hale gelen parazitoitler, içerisinde \%70'lik etil alkol bulunan eppondorf tüplerine alınmıştır. Tüpler içerisine parazitoitlere ait etiket bilgileri yazılarak teşhisleri yapılmak üzere saklanmıştır. Parazitoit türlerin teşhisi Prof. Dr. Zeljko Tomanovic (Belgrad university, Department of Invertebrate Zoology and Entomology) tarafından yapılmış ve araştırıcının koleksiyonunda saklanmaktadır.

\section{Coccinellidlerin belirlenmesi}


Kayseri ili süs bitkilerindeki yaprakbitlerinin parazitoitleri ve avc1 Coccinellidleri

Üzerinde Coccinellid larva ve yumurtaları ve yaprakbiti kolonileri bulunan bitki parçaları, Coccinellidlerin ergin döneme ulaşabilmeleri için laboratuvarda 3 lt'lik plastik kavanozların içinde ayrı ayrı kültüre alınmıştır. Plastik kavanozların üzeri bitkilerin hava almasını sağlamak amacıyla ince delikli tül ile örtülmüştür. Ergin halde laboratuvara getirilen veya daha sonra ergin hale gelen Coccinellidler ventralden üçgen şeklinde kesilmiş kartonlara yapıştırılarak ve etiket bilgileri yazılarak teşhise hazır hale getirilmiştir. Toplanan Coccinellidae familyasına ait örneklerin teşhisi Prof. Dr. Nedim UYGUN (Çukurova Üniversitesi, Bitki Koruma Bölümü, emekli öğretim üyesi) tarafından yapılmış ve araştırıcının koleksiyonunda saklanmaktadır.

\section{Konukçu Yaprakbitlerinin belirlenmesi}

Üzerinde doğal düşman saptanan yaprakbiti kolonilerine ait örnekler, laboratuvara getirildikten sonra her bir örnekten birkaç ergin yaprakbiti olmak üzere içerisinde \%70'lik alkol bulunan eppendorf tüplerine alınarak etiketlenmiștir. Yaprakbiti örneklerinin teşhisi Dr. Işıl Özdemir (Ankara Zirai Mücadele Merkez Araştırma Enstitüsü) tarafından yapılmış ve araştırıcının koleksiyonunda saklanmaktadır.

\section{Konukçu bitkilerin belirlenmesi}

Yaprakbitlerinin konukçusu olan bitkilere ait örnekler kese kağıdı içerisinde alınarak teşhisi yapılmak üzere saklanmıştır. Konukçu bitki örneklerinin teşhisleri Prof. Dr. Cem VURAL (Erciyes Üniversitesi, Biyoloji Bölümü) tarafından yapılmış ve araştırıcının koleksiyonunda saklanmaktadır.

\section{Bulgular ve tartışma}

Bu çalışma kapsamında 2014-2015 yılları arasında Kayseri ili merkez ilçelerinde park ve süs bitkilerinde beslenen yaprakbitlerinin doğal düşmanlarının tespiti amaciyla yapılan sörveylerde Aphidiinae (Hymenoptera: Braconidae) alt familyasından 9 tür tespit edilmiş, 1 parazitoit ise cins düzeyinde teşhis edilebilmiştir. Ayrıca, yaprakbitlerinin avcı Coccinellidleri olarak 11 tür belirlenmiştir. Bulunan bu türler aşağıda listelenmiştir.

\section{Parazitoit Türler}

\section{Takım Hymenoptera}

\section{Familya Braconidae}

Altfamilya Aphidiinae

Aphidius hortensis Marshall, 1896 
İncelenen materyal: Liosomaphis berberidis (Fitch, 1851), Berberis thunbergii (Berberiidaceae), Erciyes Üniversitesi Kampüsü (Melikgazi), 14.v.2015, 11우의, $2 \hat{\jmath}$.

Dünyadaki dağılımı: Palaearktik Bölge ve Nearktik Bölge'de yaygınlık gösterir (Achterberg 2017).

Türkiye'deki Dağılımı: Çanakkale (Kök et al. 2017).

Aphidius matricariae Haliday, 1834

İncelenen materyal: Macrosiphum (Macrosiphum) euphorbiae (Thomas, 1878), Rubus sp. (Rosaceae), Adnan Menderes Park1 (Melikgazi), 24.v.2014, 1 ㅇ.

Dünyadaki dağılımı: Afro-Tropikal Bölge, Avustralyan Bölge, Nearktik Bölge, Neotropikal Bölge ve Palaearktik Bölge'de dağılım gösterir (Achterberg 2017).

Türkiye'deki Dağılımı: Adana, Balıkesir, Bilecik, Bursa, Çanakkale, Çorum, Diyarbakır, Edirne, Hatay, İzmir, Kahramanmaraş, Kocaeli, Mersin, Sakarya (Erkin 1983; Karaat et al. 1986; Yumruktepe \& Uygun 1994; Uygun et al. 1995; Ölmez \& Ulusoy 2003; Ayyıldız \& Atlıhan 2006; Çetin Erdoğan et al. 2008; Çetin Erdoğan et al. 2010, Güncan et al. 2010; Kök et al. 2017).

Aphidius urticae Haliday, 1834

İncelenen materyal: Aphis (Aphis) gossypii Glover, 1854, Rubus sp., Cemilbaba Park1 (Talas), 28.v.2015, 1+; M. euphorbiae, Rubus sp., Hisarc1k Fatih Park1

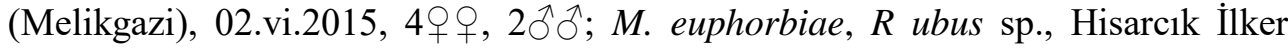
Başbuğ Park1 (Melikgazi), 23.v.2015, 2 웅, 1ð; M. euphorbiae, Rubus sp., Hisarc1k Fatih Park1 (Melikgazi), 16.vi.2015, 2우, 10․

Dünyadaki dağılımı: Palaearktik Bölge'de dağılım gösterir (Achterberg 2017).

Türkiye'deki Dağılımı: Bursa, Edirne, Erzurum, Kastamonu, Samsun, Sinop, Tekirdağ (Tomanović et al. 2008).

Aphidius rosae Haliday, 1834

İncelenen materyal: Macrosiphum (Macrosiphum) rosae (Linnaeus, 1758), Rosa

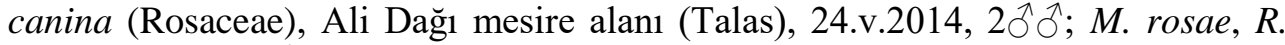
canina, Hisarcık İlker Başbuğ Parkı (Melikgazi), 25.vi.2015, 19, 1Оَ; Myzaphis rosarum (Kaltenbach, 1843), Rosa sp., Hisarcık Kayadibi camii bahçesi (Melikgazi), 27.vi.2015, 20ิ ठิ.

Dünyadaki dağ 1 lımı: Nearktik Bölge ve Palaearktik Bölge'de dağ 1 lım gösterir (Achterberg 2017).

Türkiye'deki Dağılımı: Ankara, Çanakkale, Edirne, Sinop (Düzgüneş et al. 1982; Çetin Erdoğan et al. 2010; Akar \& Çetin Erdoğan 2017; Kök et al. 2017). 
Kayseri ili süs bitkilerindeki yaprakbitlerinin parazitoitleri ve avc1 Coccinellidleri

Binodoxys angelicae (Haliday, 1833)

İncelenen materyal: Aphis (Aphis) fabae Scopoli, Viburnum opulus (Adoxaceae), 1763, Göğüs Hastalıkları Hastanesi (Melikgazi), 31.v.2015, 1 \% .

Dünyadaki dağılımı: Afro-Tropikal Bölge, Nearktik Bölge ve Palaearktik Bölge'de dağılım gösterir (Achterberg 2017).

Türkiye'deki Dağılımı: Adana, Antalya, Diyarbakır, Edirne, Hatay, İzmir, Kahramanmaraş, Mersin (Soydanbay 1976; Erkin 1983; Zeren \& Düzgüneş 1983; Yumruktepe \& Uygun 1996; Ölmez \& Ulusoy 2003; Aslan et al. 2004; Çetin Erdoğan et al. 2008; Yoldaş et al. 2011; Akar \& Çetin Erdoğan 2017).

Lysiphlebus fabarum (Marshall, 1896)

İncelenen materyal: A. fabae, Hedera helix (Araliaceae), Cumhuriyet Meydanı (Melikgazi), 20.v.2015, 1여 A. fabae, H. helix, Muhsin Yazıcıŏlu Parkı (Talas), 22.v.2015, 49우; A. fabae, V. opulus, Göğüs Hastalıkları Hastanesi (Melikgazi), 31.v.2015, 20 우우 A. fabae, V. opulus, Şelale Parkı (Hacılar), 04.vii.2015, 2 우; A. fabae, H. helix, Cumhuriyet Meydanı (Melikgazi), 03.viii.2015, 4우․

Dünyadaki dă̆ılımı: Afro-Tropikal Bölge, Nearktik Bölge ve Palaearktik Bölge'de dağılım gösterir (Achterberg 2017).

Türkiye'deki Dağılımı: Adana, Adıyaman, Ankara, Bursa, Çanakkale, Diyarbakır, Edirne, Hatay, İzmir, Kahramanmaraş, Kocaeli, Konya, Mardin, Mersin, Muğla, Sakarya, Şanlıurfa, Şırnak, Tekirdağ (Soydanbay 1976; Düzgüneş et al.1982; Erkin 1983; Karaat \& Göven 1986; Karaat et al. 1986, Kıran 1994; Özder \& Toros 1999; Ölmez \& Ulusoy 2003; Aslan et al. 2004; Güz \& K1lınçer 2005; Çetin Erdoğan et al. 2008; Akar \& Çetin Erdoğan 2017; Kök et al. 2017).

Lysiphlebus testaceipes (Cresson, 1880)

İncelenen materyal: A. fabae, Viburnum orientale (Adoxaceae), Şelale Park1 (Hac1lar), 12.vi.2014, 1 ㅇ.

Dünyadaki dağılımı: Afro-Tropikal Bölge, Nearktik Bölge, Neotropikal Bölge ve Palaearktik Bölge'de dağılım gösterir (Achterberg 2017).

Türkiye'deki Dağılımı: Aydın, Çanakkale, İzmir (Yoldaş et al. 2011; Yerlikaya et al. 2016; Kök et al. 2017).

Praon abjectum (Haliday, 1833)

İncelenen materyal: A. fabae, Philadelphus coronarius (Hydrangaceae), Cemilbaba Parkı (Talas), 05.vi.2014, 1 ㅇ.

Dünyadaki dağılımı: Nearktik Bölge, Oriental Bölge ve Palaearktik Bölge'de dağılım gösterir (Achterberg 2017). 
Türkiye'deki Dağılımı: Balıkesir, Bilecik, Bursa, Edirne (Tomanoviç et al. 2008, Akar \& Çetin Erdoğan 2017).

Praon volucre (Haliday, 1833)

İncelenen materyal: M. rosae, R. canina, Ali Dağı mesire alanı (Talas), 24.v.2014, 1 ․

Dünyadaki dağılımı: Nearktik Bölge ve Palaearktik Bölge'de dağılım gösterir (Achterberg 2017).

Türkiye'deki Dağılımı: Adana, Amasya, Ankara, Balıkesir, Bilecik, Bolu, Bursa, Çanakkale, Diyarbakır, Edirne, Erzurum, Gümüşhane, Hatay, Iğdır, İzmir, Mersin, Ordu, Samsun, Tekirdağ, Tokat (Düzgüneş et al. 1982; Zeren \& Düzgüneş 1983; Yumruktepe \& Uygun 1994; Özder \& Toros 1999; Ölmez \& Ulusoy 2003, Ayy1ldız \& Atlıhan 2006; Çetin Erdoğan et al. 2008; Daşç1 \& Güçlü 2008; Çetin Erdoğan et al. 2010; Yoldaş et al. 2011; Güçlü et al. 2015; Akar \& Çetin Erdoğan 2017).

\section{Aphidius sp.}

İncelenen materyal: M. euphorbiae, Rubus sp., Hisarcık Fatih Park1 (Melikgazi), 02.vi.2015.

\section{Avcı Coccinellid Türler}

\section{Takım Coleoptera}

\section{Familya Coccinellidae}

Adalia (Adalia) bipunctata (Linnaeus, 1758)

İncelenen materyal: A. fabae, V. opulus, Erciyes Üniversitesi kampüsü (Melikgazi), 25.v.2014 (4), 26.v.2014 (1), 27.v.2014 (1); A. fabae, P. coronarius, Meydan (Talas), 04.vi.2014 (2); A. gossypii, Rubus sp., Cemilbaba Parkı (Talas), 25.v.2014 (5); Aulacorthum (Aulacorthum) solani (Kaltenbach, 1843), V. orientale, Şelale Park1 (Hac1lar), 10.v.2014 (1); Brachycaudus (Brachycaudus) helichrysi (Kaltenbach, 1843), Cydonia sp. (Rosaceae), İnönü Park1 (Kocasinan), 28.v.2014 (1); Myzocallis (Myzocallis) coryli (Goetze,1778), Corylus avellana (Betulaceae), İnönü Park1 (Kocasinan), 26.V.2015 (1).

Dünyadaki dağılımı: Kozmopolit bir türdür (Audisio 2017).

Türkiye'deki Dağılımı: Adana, Adıyaman, Afyonkarahisar, Ankara, Artvin, Aydın, Balıkesir, Bartın, Bursa, Çanakkale, Denizli, Diyarbakır, Edirne, Elazığ, Erzincan, Erzurum, Hakkâri, Hatay, İstanbul, İzmir, Iğdır, Isparta, Kahramanmaraş, Kocaeli, Konya; Malatya, Manisa, Mardin, Mersin, Niğde, Şanlıurfa, Rize, Sakarya, Van, Yalova ve Yozgat (Giray 1970; Soydanbay 1976; Uygun 1981; Düzgüneş et al. 1982; Erkin 1983; Özbek \& Çetin, 1991; Yumruktepe \& Uygun 1994; Uygun et al. 1995; Ulusoy et al. 1999; Yaşar et al. 1999; Yurtsever 2001; Tezcan \& Uygun 2003; 
Kayseri ili süs bitkilerindeki yaprakbitlerinin parazitoitleri ve avc1 Coccinellidleri

Öztürk et al. 2004; Aslan \& Uygun 2005; Bolu 2005; Gençer et al. 2005; Karaca et al. 2006; Şahbaz \& Uysal 2006; Bolu et al. 2007; Hepdurgun et al. 2007; Daşc1 \& Güçlü 2008; Narmanlıoğlu \& Güçlü 2008; Yeşilayer 2009; Güncan et al. 2010; Portakaldalı \& Satar 2010; Başar \& Yaşar 2011; Kaya \& Yaşar, 2011; Gözüaçık et al. 2012; Varlı et al., 2013; Demirözer \& Karaca 2014; Baştuğ \& Kasap 2015; Buğday et al. 2015; Yoldaş et al. 2011; Varlı et al. 2013; Sobutay 2016; Yerlikaya et al. 2016; Kök et al. 2017; Tiftikci 2017).

Adalia (Adalia) decempunctata (Linnaeus, 1758)

İncelenen materyal: A. fabae, V. opulus, Erciyes Üniversitesi kampüsü (Melikgazi), 25.v.2014 (1).

Dünyadaki dağılımı: Palaearktik Bölge'de dağılım gösterir (Audisio 2017).

Türkiye'deki Dağılımı: Adana, Ankara, Artvin, Balıkesir, Bartın, Bursa, Çanakkale, Diyarbakır, Edirne, Elazığ, Hatay, İstanbul, İzmir, Iğdır, Isparta, Kahramanmaraş, Konya, Manisa, Mardin, Mersin, Niğde, Rize, Yalova, Yozgat (Soydanbay 1976; Uygun 1981; Düzgüneş et al. 1982; Erkin 1983; Yumruktepe \& Uygun 1994; Ulusoy et al. 1999; Yurtsever 2001; Tezcan \& Uygun 2003; Aslan \& Uygun 2005; Bolu 2005; Karaca et al. 2006; Şahbaz \& Uysal 2006; Bolu et al. 2007; Hepdurgun et al. 2007; Güncan et al. 2010; Başar \& Yaşar 2011; Kaya \& Yaşar, 2011; Keskin 2012; Varlı et al., 2013; Baştuğ \& Kasap 2015; Buğday et al. 2015; Sobutay 2016; Kök et al. 2017; Tiftikci 2017).

\section{Adalia fasciatopunctata revelierei (Mulsant, 1866)}

İncelenen materyal: A. solani, V. orientale, Şelale Park1 (Hac1lar), 10.v.2014 (5); B. helichrysi, Cydonia sp., Adnan Menderes Park1 (Melikgazi), 28.v.2014 (3); Aphis (Aphis) craccivora Koch, 1854, Rubus sp., Adnan Menderes Park1 (Melikgazi), 24.v.2014 (1); A. craccivora, Salix sp., Adnan Menderes Park1 (Melikgazi), 24.v.2014 (1); A. fabae, V. opulus, Erciyes Üniversitesi kampüsü (Melikgazi), 25.v.2014 (7); M. coryli, C. avellana, Erciyes Üniversitesi kampüsü (Melikgazi), 26.v.2015 (2); M. euphorbiae, B. thunbergii, Talas Erkek Yurdu (Talas), 08.vi.2014 (3); A. fabae, P. coronarius, Cemilbaba Park1 (Talas), 04.vi.2014 (6), 13.vi.2015 (2); A. gossypii, Rubus sp., Cemilbaba Park1 (Talas), 04.vi.2014 (2).

Dünyadaki dağılımı: Palaearktik Bölge'de dağılım gösterir (Horion 1961).

Türkiye'deki Dağılımı: Adana, Ankara, Artvin, Bartın, Balıkesir, Burdur, Bursa, Çanakkale, Denizli, Diyarbakır, Elazığ, Erzincan, Erzurum, Hakkari, Hatay, İzmir, İstanbul, Iğdır, Isparta, Kahramanmaraş, Kars, Konya, Manisa, Mersin, Tekirdağ, Van, Yalova, Yozgat, (Giray 1970; Düzgüneş et al. 1982; Uygun 1981; Özbek \& Çetin, 1991; Elmalı \& Toros 1994; Yumruktepe \& Uygun 1994; Özder \& Toros 1999; Yaşar et al. 1999; Tezcan \& Uygun 2003; Aslan \& Uygun 2005; Karaca et al. 2006; Şahbaz \& Uysal 2006; Bolu et al. 2007; Hepdurgun et al. 2007; Bayram 2008; 
Bayram 2009; Daşc1 \& Güçlü 2008; Yeşilayer 2009; Kaya \& Yaşar, 2011; Demirözer \& Karaca 2014; Baştuğ \& Kasap 2015; Buğday et al. 2015; Kaplan \& Turanlı 2016; Kütük \& Güçlü 2016; Sobutay 2016; Kök et al. 2017; Tiftikci 2017).

Chilocorus bipustulatus (Linnaeus, 1758)

İncelenen materyal: Cinara (Cubressobium) cupressi (Buckton, 1881), Thuja sp. (Cupressaceae), Erciyes Üniversitesi Kampüsü (Melikgazi), 30.viii.2014 (1).

Dünyadaki dağılımı: Palaearktik Bölge'de dağılım gösterir (Audisio 2017).

Türkiye'deki Dağılımı: Adana, Afyon, Ankara, Antalya, Artvin, Aydın, Balıkesir, Çanakkale, Denizli, Diyarbakır, Elazı ̆̆, Erzincan, Gaziantep, Hakkâri, Hatay, Isparta, İzmir, Manisa, Mardin, Mersin, Muğla, Kahramanmaraş, Siirt, Şanlıurfa, Van (Soydanbay 1976; Uygun 1981; Karaat \& Güven 1986; Yumruktepe \& Uygun 1994; Erol \& Yaşar 1996; Yaşar et al. 1999; Erler \& Tunç 2001; Ülgentürk et al. 2004; Bolu et al. 2005; Özgen \& Karsavuran 2005; Bolu et al. 2007; Hepdurgun et al. 2007; Kaya \& Yaşar 2011; Hazır \& Ulusoy 2012; Keskin 2012; Varlı et al. 2013; Demirözer \& Karaca 2014; Baştuğ \& Kasap 2015; Çulcu \& Mart 2015; Kaplan \& Turanlı 2016; Kütük \& Güçlü 2016; Kök et al. 2017).

Coccinella (Coccinella) septempunctata Linnaeus, 1758

İncelenen materyal: A. fabae Scopoli, V. opulus, Şelale Parkı (Hacılar), 04.vii.2015 (3).

Dünyadaki dağılımı: Nearktik Bölge, Oriental Bölge ve Palaearktik Bölge'de dağılım gösterir (Audisio 2017).

Türkiye'deki Dağılımı: Adana, Adıyaman, Ankara Aydın, Balıkesir, Bursa, Denizli, Diyarbakır, Edirne, Elazığ, Erzurum, Erzincan, Gaziantep, Hatay, Isparta, İzmir, Kars, Kahramanmaraş, Konya, Mardin, Mersin, Siirt, Şanlıurfa, Tekirdağ, Van, Yalova ve Yozgat (Soydanbay 1976; Uygun 1981; Karaat \& Güven 1986; Kaya \& Hıncal 1991; Özbek \& Çetin 1991; Elmalı \& Toros 1994; Yumruktepe \& Uygun 1994; Özder \& Toros 1999; Yaşar et al. 1999; Yurtsever 2001; Sertkaya \& Yiğit 2002; Atlıhan \& Özgökçe 2003; Aslan \& Uygun 2005; Bolu et al. 2005; Çetin \& Alaoğlu 2005; Gençer et al. 2005; Özkan et al. 2005; Ayyıldız \& Atlıhan 2006; Işıkber \& Karcı 2006; Karaca et al. 2006; Şahbaz \& Uysal 2006; Elekçioğlu \& Şenal 2007; Daşc1 \& Güçlü 2008; Bayram 2009; Güncan et al. 2010; Kaya \& Yaşar 2011; Yoldaş et al. 2011; Gözüaçık et al. 2012; Hazır \& Ulusoy 2012; Keskin 2012; Varlı et al. 2013; Demirözer \& Karaca 2014; Baştuğ \& Kasap 2015; Buğday et al. 2015; Çulcu \& Mart 2015; Kaplan \& Turanl1 2016; Kütük \& Güçlü 2016; Sobutay 2016; Yerlikaya et al. 2016; Kök et al. 2017; Tiftikci 2017).

Exochomus (Exochomus) quadripustulatus Linnaeus, 1758 
Kayseri ili süs bitkilerindeki yaprakbitlerinin parazitoitleri ve avc1 Coccinellidleri

İncelenen materyal: Eulachnus rileyi (Williams, 1911), Pinus sp. (Pinaceae), Şelale Park1 (Hacilar), 10.v.2014 (1); B. helichrysi, Sorbus sp. (Rosaceae), Erciyes Üniversitesi Kampüsü (Melikgazi), 31.v.2014 (2).

Dünyadaki dağılımı: Nearktik Bölge ve Palaearktik Bölge'de dağılım gösterir (Audisio 2017).

Türkiye'deki Dağılımı: Adana, Adıyaman, Ankara, Artvin, Balıkesir, Çanakkale, Denizli, Diyarbakır, Edirne, Elazığ, Erzincan, Erzurum, Hatay, İstanbul, İzmir, Iğdır, Isparta, Kahramanmaraş, Konya, Manisa, Mardin, Mersin, Muğla, Niğde, Rize, Siirt, Şanlıurfa, Van, Yalova ve Yozgat (Giray 1970; Soydanbay 1976; Düzgüneş et al. 1982; Özbek \& Çetin 1991; Yumruktepe \& Uygun 1994; Ulusoy et al. 1999; Yaşar et al. 1999; Yurtsever 2001; Tezcan \& Uygun 2003; Ülgentürk et al. 2004; Aslan \& Uygun 2005; Bolu 2005; Özgen \& Karsavuran 2005; Karaca et al. 2006; Şahbaz \& Uysal 2006; Bolu et al. 2007; Hepdurgun et al. 2007; Bayram 2008; Daşc1 \& Güçlü 2008; Bayram 2009; Yeşilayer 2009; Portakaldalı \& Satar 2010; Başar \& Yaşar 2011; Kaya \& Yaşar 2011; Gözüaçık et al. 2012; Keskin 2012; Demirözer \& Karaca 2014; Baştuğ \& Kasap 2015; Buğday et al. 2015; Kütük \& Güçlü 2016; Tiftikci 2017).

\section{Hippodamia (Hippodamia) variegata Goeze, 1777}

İncelenen materyal: A. gossypii, Hibiscus syriacus (Malvaceae), Çorakçılar Parkı (Melikgazi), 20.viii.2014 (1); A. pomi, Photinia sp. (Rosaceae), İnönü Park1 (Kocasinan), 30.v. 2014 (1); A. pomi, Photinia sp. Halef hoca Mezarlığı (Talas), 31.v.2014 (1); A. solani, V. opulus, Şelale Park1 (Hacilar), 25.vi.2014 (3), 25.viii.2014 (2).

Dünyadaki dağılımı: Afro-Tropikal Bölge, Nearktik Bölge, Oriental Bölge ve Palaearktik Bölge'de dağılım gösterir (Audisio 2017).

Türkiye'deki Dağılımı: Adana, Adıyaman, Ankara, Amasya, Antalya, Artvin (Şavşat), Aydın, Balıkesir, Burdur, Çanakkale, Diyarbakır, Edirne, Elazığ, Erzurum, Erzincan, Gaziantep, Hatay, Isparta, İzmir, Kahramanmaraş, Kars, Manisa, Mardin, Mersin, Siirt, Şanlıurfa, Tekirdağ, Van, Yalova ve Yozgat (Uygun 1981; Özbek \& Çetin 1991; Yumruktepe \& Uygun 1994; Tamer et al. 1997; Özder \& Toros 1999; Yaşar et al. 1999; Yurtsever 2001; Atlıhan \& Özgökçe 2003; Tezcan \& uygun 2003; Gençsoylu \& Yalçın 2004; Öztürk et al. 2004; Aslan \& Uygun 2005; Bolu et al. 2005; Özgen \& Karsavuran 2005; Ayyıldız \& Atlıhan 2006; Işıkber \& Karcı 2006; Şahbaz \& Uysal 2006; Bolu et al. 2007; Hepdurgun et al. 2007; Daşc1 \& Güçlü 2008; Yeşilayer 2009; Güncan et al. 2010; Portakaldalı \& Satar 2010; Başar \& Yaşar 2011; Kaya \& Yaşar 2011; Yoldaş et al. 2011; Gözüaçık et al. 2012; Keskin 2012; Varlı et al. 2013; Bali et al. 2014; Demirözer \& Karaca 2014; Baştuğ \& Kasap 2015; Buğday et al. 2015; Çulcu \& Mart 2015; Kaplan \& Turanlı 2016; Kütük \& Güçlü 2016; Kök et al. 2017; Tiftikci 2017). 
Oenopia conglobata (Linnaeus, 1758)

İncelenen materyal: B. helichrysi, Photinia sp., İnönü Park1 (Kocasinan), 28.v.2014 (1); B. helichrysi, Sorbus sp., Erciyes Üniversitesi Kampüsü (Melikgazi), 28.v.2014 (1); C. cupressi, Thuja sp., İncesu park1 (İncesu), 10.v.2014 (1); Dysaphis (Dysaphis) crataegi (Kaltenbach, 1843), Crataegus sp. (Rosaceae), Kıranard1 (Melikgazi), 24.v.2014 (1).

Dünyadaki dağılımı: Palaearktik Bölge'de dağılım gösterir (Audisio 2017).

Türkiye'deki Dağılımı: Adıyaman, Ankara, Antalya, Aydın, Balıkesir, Bartın, Çanakkale, Diyarbakır, Elazığ, Erzincan, Erzurum, Gaziantep, Kahramanmaraş, Manisa, Mardin, Iğdır, Isparta, İzmir, Rize, Siirt, Şanlıurfa, Yalova ve Yozgat illerinde bulunduğu (Giray 1970; Özbek \& Çetin 1991; Tezcan \& uygun 2003; Aslan \& Uygun 2005; Özgen \& Karsavuran 2005; Ayyıldız \& Atlıhan 2006; Karaca et al. 2006; Şahbaz \& Uysal 2006; Bolu et al. 2007; Hepdurgun et al. 2007; Daşc1 \& Güçlü 2008; Bayram 2009; Güncan et al. 2010; Portakaldalı \& Satar 2010; Başar \& Yaşar 2011; Yoldaş et al. 2011; Gözüaçık et al. 2012; Hazır \& Ulusoy 2012; Keskin 2012; Varlı et al. 2013; Bali et al. 2014; Baştuğ \& Kasap 2015; Buğday et al. 2015; Çulcu \& Mart 2015; Kaplan \& Turanlı 2016; Kütük \& Güçlü 2016; Sobutay 2016; Yerlikaya et al. 2016; Kök et al. 2017; Tiftikci 2017).

Propylea quatuordecimpunctata (Linnaeus, 1758)

İncelenen materyal: A. solani, V. opulus, Şelale Parkı (Hacılar), 25.vi.2014 (1).

Dünyadaki dağılımı: Nearktik Bölge ve Palaearktik Bölge'de dağılım gösterir (Audisio 2017).

Türkiye'deki Dă̆ılımı: Adana, Balıkesir, Bartın, Çanakkale, Denizli, Edirne, Erzurum, Erzincan, Hatay, Iğdır, Isparta, İzmir, Kahramanmaraş, Kars, Konya, Manisa, Mersin, Van, Yalova (Giray 1970; Soydanbay 1976; Özbek \& Çetin 1991; Yumruktepe \& Uygun 1994; Yaşar et al. 1999; Yurtsever 2001; Atlıhan \& Özgökçe 2003; Aslan \& Uygun 2005; Ayyıldız \& Atlıhan 2006; Şahbaz \& Uysal 2006; Daşc1 \& Güçlü 2008; Kaya \& Yaşar 2011; Yoldaş et al. 2011; Keskin 2012; Baştuğ \& Kasap 2015; Buğday et al. 2015; Kaplan \& Turanlı 2016; Sobutay 2016).

Scymnus (Scymnus) apetzi Mulsant, 1846

İncelenen materyal: Mindarus abietinus Koch, 1857, Cedrus sp. (Pinaceae), Feyyaz Mercan İlkokulu (Hac1lar), 30.viii.2014 (1).

Dünyadaki dağılımı: Palaearktik Bölge'de dağılım gösterir (Audisio 2017).

Türkiye'deki Dağılımı: Adana, Adıyaman, Ankara, Balıkesir, Çanakkale, Erzurum, Erzincan, Gaziantep, Hatay, Isparta, İzmir, Kahramanmaraş, Mersin, Siirt, Şanlıurfa, Van, Yozgat illerinde bulunduğu (Soydanbay 1976; Düzgüneş et al. 1982; Özbek \& Çetin 1991; Yumruktepe \& Uygun 1994; Yaşar et al. 1999; Atlıhan \& Özgökçe 
Kayseri ili süs bitkilerindeki yaprakbitlerinin parazitoitleri ve avc1 Coccinellidleri

2003; Aslan \& Uygun 2005; Ayyıldız \& Atlıhan 2006; Bolu et al. 2007; Hepdurgun et al. 2007; Bayram 2008; Başar \& Yaşar 2011; Kaya \& Yaşar 2011; Çulcu \& Mart 2015; Tiftikci 2017).

\section{Scymnus (Pullus) subvillosus (Goeze, 1777)}

İncelenen materyal: Cinara (Cinara) cedri Mimeur, 1936, Cedrus sp., Erciyes Üniversitesi Kampüsü (Melikgazi), 15.viii.2014 (1).

Dünyadaki dağılımı: Palaearktik Bölge'de dağılım gösterir (Audisio 2017).

Türkiye'deki Dağılımı: Adana, Adıyaman, Ankara, Aydın, Balıkesir, Çanakkale, Diyarbakır, Elazı ğ, Gaziantep, Hatay, Isparta, İzmir, Kahramanmaraș, Konya, Mardin, Mersin, Siirt, Şanluurfa, Van, Yalova (Soydanbay 1976; Yumruktepe \& Uygun 1994; Yaşar et al. 1999; Aslan \& Uygun 2005; Özgen \& Karsavuran 2005; Ayyıldız \& Atlıhan 2006; Şahbaz \& Uysal 2006; Bolu et al., 2007; Hepdurgun et al. 2007; Bayram 2009; Kaya \& Yaşar 2011; Gözüaçık et al. 2012; Varlı et al. 2013; Baştuğ \& Kasap 2015; Buğday et al. 2015; Çulcu \& Mart 2015; Kök et al. 2017).

Çalışmada, Kayseri ili merkez ilçelerinde süs bitkilerinde zararlı yaprakbiti türlerinin parazitoit ve avcı Coccinellidleri saptanmıştır. Yapılan literatür incelemesinde daha önce Kayseri'de yaprakbitlerinin parazitoitleri veya avc1 Coccinellidleri ile ilgili bir çalışmaya rastlanılmadığı için çalışmada saptanan türlerin hepsinin Kayseri ili için yeni kayıt niteliğinde olduğu düşünülmektedir. Bununla birlikte, çalışmada yaprakbiti parazitoitlerinin bazı hiperparazitoitleri ve diğer takım ve familyalardan avcıları da elde edilmiş ancak bu türler teșhis edilememiștir.

Yapılan sörveyler sırasında Kayseri ili merkez ilçelerinde özellikle parklarda oldukça yoğun bir ilaçlama programının uygulandığı gözlemlenmiştir. Yapılan yoğun ilaçlamaların zararlılar ile birlikte zararlıların doğal düşmanlarını da olumsuz yönde etkilediği yadsınamaz bir gerçektir. Bu nedenle, süs bitkilerinde bulunan zararlılara karşı ilaçlama gerekip gerekmediği dikkatle incelenmeli, eğer ilaçlama gerekiyorsa ilaçlamaların zamanının, sayısının ve dozunun, mutlaka çevre sağlığı ve doğal düşman varlığını da gözeterek planlanması gerekmektedir. Bu şekilde, gerek çevre sağlığı, gerek ekonomik olarak ve gerekse bu alanlardaki biyoçeşitliliğin korunması açısından önemli kazanımlar elde edilebilecektir. Ayrıca böyle bir yaklaşım sonucunda, doğal düşman popülâsyonlarının yaprakbiti popülâsyonlarını uzun vadede daha başarılı bir şekilde baskı altında tutabileceği düşünülmektedir.

\section{Teşekkür}

Çalışmada elde edilen parazitoit türlerin teşhisini yapan Prof. Dr. Zeljko Tomanovic (Belgrad university, Department of Invertebrate Zoology and Entomology)'a, Coccinellidlerin teşhisini yapan Prof. Dr. Nedim UYGUN (Çukurova Üniversitesi, Bitki Koruma Bölümü, emekli öğretim üyesi)'a, Yaprakbitlerinin teşhisini yapan Dr. Iş1l Özdemir (Ankara Zirai Mücadele Merkez Araştırma Enstitüsü)'e ve Konukçu bitkilerin teşhislerini yapan Prof. Dr. Cem VURAL (Erciyes Üniversitesi, Biyoloji 58 
Bölümü)'a ve Bu çalışmayı FYL-2014-5341 numaralı proje ile destekleyen Erciyes Üniversitesi Bilimsel Araştırma Projeleri Birimi’ne içtenlikle teşekkür ederiz.

\section{Kaynaklar}

Achterberg C van 2017. Fauna Europaea: Hymenoptera: Braconidae: Aphidiinae. Fauna Europaea version 2017.06. URL: https://fauna-eu.org (Erişim tarihi:09 Şubat 2018).

Akar S. \& Ö. Çetin Erdoğan 2017. Contributions to Aphidiinae (Hymenoptera: Braconidae) fauna of Turkey with new records. Trakya University Journal of Natural Sciences, 18(2): 89-96.

Aslan M.M., N. Uygun \& P. Starý 2004. A survey of aphid parasitoids in Kahramanmaras, Turkey (Hymenoptera: Braconidae, Aphidiinae; and: Aphelinidae). Phytoparasitica, 32: 255-263.

Aslan M.M. \& N. Uygun 2005. The aphidophagus Coccinellid (Coleoptera: Coccinellidae) species in Kahramanmaraş, Turkey. Turkish Journal of Zoology, 29:1-8.

Atlıhan R. \& M.S. Özgökçe 2003. Van ili şekerpancarı alanlarındaki zararlı ve yararlı türlerin saptanması. Yüzüncü Yll Üniversitesi Ziraat Fakültesi Tarım Bilimleri Dergisi, 13 (1): 914 .

Audisio P. 2017. Fauna Europaea: Coleoptera: Coccinellidae. Fauna Europaea version 2017.06. URL: https://fauna-eu.org (Erişim tarihi:09 Şubat 2018).

Ayyıldız Y. \& R. Atlıhan 2006. Balıkesir ili sebze alanlarında görülen yaprakbiti türleri ve doğal düşmanları. Yüzüncü Yıl Üniversitesi Ziraat Fakültesi Tarım Bilimleri Dergisi, 16 (1): $1-5$

Bali B., S.M. Özgökçe \& D. Şenal 2014. Antalya ve çevresinde Coccinellidae (Coleoptera) familyasına bağlı türler ve yayılış alanları. Türkiye 5. Bitki Koruma Kongresi Bildirileri, 3-5 Şubat 2014, Antalya, 75.

Başar M. \& B. Yaşar 2011. Isparta ili meyve bahçelerinde saptanan Coccinellidae (Coleoptera) türleri. Türkiye Entomoloji Dergisi, 35(3):519-534.

Baştuğ G. \& İ. Kasap 2015. Çanakkale ili Coccinellidae (Coleoptera) familyası üzerine faunistik çalışmalar. Türkiye Biyolojik Mücadele Dergisi, 6 (1):41-50.

Bayram Ş. 2008. Ankara'da Karaağaç (Ulmus glabra Mill.)'da gal yapan yaprakbitlerinde avc1 Coccinellidae (Coleoptera), Chrysopidae ve Hemerobiidae (Neuroptera) familyasına bağlı türler. Tarım Bilimleri Dergisi, 14 (4) 386-393.

Bayram Ş. 2009. Ankara'da Elma Kırmızı Gal Yaprakbiti, Dysaphis devecta Walk. (Homoptera, Aphididae)'da avc1 Coccinellidae (Coleoptera) türleri. Tarım Bilimleri Dergisi, 15(1) 53-57.

Boivin G., T. Hance \& J. Brodeur 2012. Aphid parasitoids in biological control. Canadian Journal of Plant Science, 92 (1): 1-12.

Bolu H. 2005. On the Coccinellid (Col.) fauna of almond orchards in South Eastern and Eastern Anatolia. Zoology in the Middle East, 35:109-110.

Bolu H., İ. Özgen, A. Bayram \& M. Çınar 2007. Güneydoğu ve Doğu Anadolu Bölgelerinde Antepfistığı, Badem ve Kiraz bahçelerindeki avcı Coccinellidae türleri, yayıııı̧ alanları ve avlar1. Harran Üniversitesi Ziraat Fakültesi Dergisi, 11 (12): 39-47.

Buğday H., D. Şenal \& R. Atlıhan 2015. Yalova ilinde farklı habitatlarda bulunan Coccinellidae (Coleoptera) türleri ve yayılış alanları. Türkiye Biyolojik Mücadele Dergisi, 6 (2): 127-138. 
Kayseri ili süs bitkilerindeki yaprakbitlerinin parazitoitleri ve avc1 Coccinellidleri

Çetin Erdoğan Ö., Ž. Tomanović \& A. Beyarslan 2008. New aphid parasitoids (Hymenoptera: Braconidae: Aphidiinae) in the region of Marmara. Acta Entomologica Serbica, 13: 85- 88 .

Çetin Erdoğan Ö., Ž. Tomanović \& A. Beyarslan, 2010. New distributional records on the subfamily Aphiidinae (Hymenoptera: Braconidae) in Black Sea Region, Turkey. Linzer Biologische Beitrage, 42: 613-616.

Çetin H. \& Ö. Alaoğlu 2005. Mut (Mersin) ilçesinde zeytin ağaçlarında bulunan yararlı böcek türlerinin tespiti ve önemli türlerin populasyon değişimi. Süleyman Demirel Üniversitesi Ziraat Fakültesi Dergisi, 19 (36): 59-65.

Çulcu M. \& C. Mart 2015. Gaziantep ve Şanlıurfa illerinde Pistacia spp. yapraklarında gal oluşturan Yaprakbiti türleri, yayılış alanları ve doğal düşmanları. Bitki Koruma Bülteni, 55 (3): 171-185.

Daşcı E. \& Ş. Güçlü 2008. Iğdır ovasında meyve ağaçlarında bulunan yaprakbiti türleri (Homoptera: Aphididae) ve doğal düşmanları. Atatürk Üniversitesi Ziraat Fakültesi Dergisi, 39 (1), 71-73.

Demirözer O. \& İ. Karaca 2014. Isparta ili yağ gülü (Rosa damascena Miller) üretim alanlarında bulunan avcı ve parazitoit türler ve yayılışları. Türkiye Entomoloji Bülteni, 2014, 4 (3): 171-184

Düzgüneş Z., S. Toros, N. Kılınçer \& B. Kovancı 1982. Ankara ilinde Bulunan Aphidoidea Türlerinin parazit ve predatörlerinin tespiti. Tarım ve Orman Bakanlığı, Zirai Mücadele ve Zirai Karantina Genel Müdürlüğü Yayınları, 251 s.

Elekçioğlu N.Z. \& D. Şenal 2007. Pest and natural enemy fauna in organic citrus production in the Eastern Mediterranean region of Turkey. International Journal of Natural and Engineering Sciences, 1: 29-34.

Elmalı M. \& S. Toros 1994. Konya ilinde buğday tarlalarında yaprakbiti doğal düşmanlarının tespiti üzerinde araştırmalar. Türkiye 3. Biyolojik Mücadele Kongresi Bildirileri, 25-28 Ocak 1994, İzmir, 13-29.

Erkin E. 1983. Investigations on the hosts distribution and efficiency of the natural enemies of the family Aphididae (Homoptera) harmful to pome and stone fruits trees in Izmir province of Aegean Region. Türkiye Bitki Koruma Dergisi, 7 (1): 29-49.

Erler F. \& İ. Tunç 2001. A survey (1992-1996) of natural enemies of diaspididae species in Antalya, Turkey. Phytoparasitica, 29 (4): 299-305.

Erol T. \& B. Yaşar 1996. Van ili elma bahçelerinde bulunan zararlı türler ile doğal düşmanları. Türkiye Entomoloji Dergisi, 20 (4): 281-293.

Gençer N.S., K.S. Coşkuncu \& N.A. Kumral 2005. Bursa ilinde incir bahçelerinde görülen zararlı ve yararlı türlerin saptanması. Ondokuz Mayls Üniversitesi Ziraat Fakültesi Dergisi, 20 (2): 24-30.

Gençsoylu İ. \& İ. Yalçın 2004. The effect of different tillage systems on cotton pests and predators in cotton fields. Asian Journal of Plant Sciences, 3 (1): 39-44.

Giray H. 1970. Harmful and useful species of Coccinellidae (Coleoptera) from Aegean Region with notes on their localities, collecting dates and hosts. Year-book of the Faculty of Agriculture of Ege University, 1(1):35-50.

Gözüaçık C., A. Yiğit, N. Uygun 2012. Güneydoğu Anadolu Bölgesi'nde farklı habitatlarda bulunan Coccinellidae (Coleoptera) türleri. Türkiye Biyolojik Mücadele Dergisi, 3 (1): 69-88. 
Güçlü Ş., H. Kavaz, C. Güçlü \& I. Özdemir 2015. Aphids (Hemiptera: Aphididae) and their parasitoids on ornamental trees and shrubs in Erzurum, Turkey. Türkiye Entomoloji Dergisi, 39 (1): 3-9.

Güncan A., Z. Yoldaş \& N. Madanlar 2010. İzmir'de şeftali bahçelerinde bulunan yaprakbiti türleri ve doğal düşmanları üzerinde araştırmalar. Türkiye Entomoloji Dergisi, 34 (3): 399-408.

Güz N. \& N. Kılınçer 2005. Aphid parasitoids (Hymenoptera: Braconidae: Aphidiinae) on weeds from Ankara, Turkey. Phytoparasitica, 33 (4): 359-366.

Hazır A. \& M.R. Ulusoy 2012. Adana ve Mersin illeri şeftali ve nektarin alanlarında saptanan zararlılar ile predatör ve parazitoit türler. Türkiye Biyolojik Mücadele Dergisi, 3 (2): $157-$ 168.

Hepdurgun B., T. Turanlı, N. Uygun \& C. Kaplan 2007. Balıkesir ve Çanakkale illerinde zeytin bahçelerinde bulunan Coccinellidae türleri. Türkiye 2. Bitki Koruma Kongresi Bildirileri, Isparta, 164s.

Horion A. 1961. Faunistif der Mitteleuropäischen Käfer. Band VIII. Überlingen-Bodensee, Komissionsverlag Buchdruckerei Ang. Feysel., 283-365.

Iperti G. 1999. Biodiversity of Predaceous coccinellidae in relation to bioindication and economic importance. Agriculture, Ecosystems and Environment, 74: 323-342.

Işıkber A.A. \& A. Karcı 2006. Kahramanmaraş ili ve çevresinde bazı tarla kültürlerinde bulunan avcı böcek türlerinin yoğunluk ve yaygınlıklarının saptanması. Kahramanmaraş Sütçü Imam Üniversitesi Fen ve Mühendislik Dergisi, 9 (1): 111-116.

Kaplan C. \& T. Turanlı 2016. İzmir ve Manisa illeri kestane alanlarında saptanan Coccinellidae (Coleoptera) türleri. Türkiye Biyolojik Mücadele Dergisi, 7 (1): 65-78.

Karaat Ş. \& M.A. Göven 1986. Güneydoğu Anadolu Bölgesinde tütün dikim alanlarında şeftali yaprakbiti (Mysuz persicae Sulz.)'nin doğal düşmanlarının genel durumu. Türkiye I. Biyolojik Mücadele Kongresi Bildirileri, 12-14 Şubat 1986, Adana, 162-172.

Karaat Ş., M.A. Göven \& C. Mart 1986. Güneydogu Anadolu Bölgesi'nde pamuk dikim alanlarında yararlı türlerin genel durumları. Türkiye I. Biyolojik Mücadele Kongresi Bildirileri, 12-14 Şubat 1986, Adana, 173-185.

Karaca İ., Y. Karsavuran, M. Avc1, O. Demirözer, B. Aslan, E. Sökeli \& H.S. Bulut 2006. Isparta ilinde Coleoptera takımına ait türler üzerinde faunistik çalışmalar. Süleyman Demirel Üniversitesi Fen Bilimleri Enstitüsü Dergisi, 10 (2): 180-184.

Kaya, M. \& B. Yaşar 2011. Isparta ili meyve bahçelerinde saptanan Coccinellidae (Coleoptera) türleri. Türkiye Entomoloji Dergisi, 35 (3): 519-534.

Kaya N. \& P. Hıncal 1991. Denizli ili mercimek alanlarında bulunan böcek faunası. Türkiye Entomoloji Dergisi, (3): 173-181.

Keskin N. 2012. Bornova (İzmir) ilçesinde peyzaj alanlarındaki Coccinellidae (Coleoptera: Insecta) faunası. Yüksek Lisans Tezi, Selçuk Üniversitesi Fen Bilimleri Enstitüsü, Konya, 48s.

Kıran E. 1994. Güneydoğu Anadolu Bölgesi hububat ekiliş alanlarında görülen yaprakbiti türleri ve doğal düşmanları üzerinde çalışmalar. Türkiye 3. Biyolojik Mücadele Kongresi Bildirileri, 25-28 Ocak 1994, İzmir. 29-35.

Kök Ş., Ž. Tomanović, D. Şenal, G. Baştuğ \& İ. Kasap 2017. Aphidophagous coccinellid and parasitoid species determined in the Çanakkale Province with a new record for the parasitoid fauna of Turkey. Bitki Koruma Bülteni, 57(4): 485-502. 
Kayseri ili süs bitkilerindeki yaprakbitlerinin parazitoitleri ve avc1 Coccinellidleri

Kütük Y. \& Ş. Güçlü 2016. Erzincan ilinde kirazlarda (Prunus avium L.) zarar yapan Aphididae (Hemiptera) türleri ile parazitoit ve predatörlerinin belirlenmesi. Bitki Koruma Bülteni, 56 (2): 155-163.

Muratori F.B., R.J. Gagne \& R.H. Messing 2009. Ecological traits of a new aphid parasitoid, Endaphis fugitiva (Diptera: Cecidomyiidae), and its potential for biological control of the banana aphid, Pentalonia nigronervosa (Hemiptera: Aphididae). Biological Control, 50: 185193.

Narmanlığlu H.K. \& Ş. Güçlü 2008. İspir (Erzurum) ilçesinde meyve ağaçlarında bulunan yaprakbiti türleri (Hemiptera: Aphidiade) ve doğal düşmanları. Atatürk Üniversitesi Ziraat Fakültesi Dergisi, 39 (2): 225-229.

Oğuzoğlu Ş., M. Avc1, D. Şenal \& İ. Karaca 2017. First record of Anatis ocellata (Linnaeus, 1758) (Coleoptera: Coccinellidae) in Turkey. Türkiye Entomoloji Bülteni, 7 (2): 197-202.

Ölmez S. \& M.R. Ulusoy 2003. A survey of aphid parasitoids (Hymenoptera: Braconidae: Aphidiinae) in Diyarbakır, Turkey. Phytoparasitica, 31:524-528. 20.

Özbek H. \& G. Çetin 1991. Contribution to the fauna of coccinellidae (Coleoptera) from Eastern Anatolia Along with some new records from Turkey. Türkiye Entomoloji Dergisi, 15 (4): 193-202.

Özder N. \& S. Toros 1999. Trakya ilinde buğdaylarda zarar yapan yaprakbiti türlerinin doğal düşmanları üzerinde araştırmalar. Türkiye 4. Biyolojik Mücadele Kongresi Bildirileri. 2629 Ocak 1999, Adana, 501-512.

Özgen İ. \& Y. Karsavuran 2005. Antepfıstığı ağaçlarında zararlı Lepidosaphes pistaciae (Archangelskaya) (Homoptera: Diaspididae)'nin doğal düşmanlarının saptanması üzerinde araştırmalar. Türkiye Entomoloji Dergisi, 29 (4): 309-316.

Özkan C., O. Gürkan \& Ö. Hancıoğlu 2005. Çubuk (Ankara) ilçesi vişne ağaçlarında zararlı olan türler, doğal düşmanları ve önemlileri üzerinde gözlemler. Tarım Bilimleri Dergisi, 11 (1): 57-59.

Öztürk N., M.R. Ulusoy, L. Erkılıç, S. Bayhan 2004. Malatya ili kayısı bahçelerinde saptanan zararlılar ile avcı türler. Bitki Koruma Bülteni, 44 (1-4): 1-13.

Portakaldalı M. \& S. Satar 2010. Artvin ve Rize illeri Coccinellidae (Coleoptera) faunası üzerinde çalışmalar. Bitki Koruma Bülteni, 50 (3): 89-99.

Sertkaya E. \& A. Yiğit 2002. Antakya ve çevresinde buğdayda zararlı yaprakbiti türleri ve doğal düşmanları. Türkiye 5. Biyolojik Mücadele Kongresi Entomoloji Derneği Yayınlar1, 4-7 Eylül 2002, Erzurum, 265-274.

Smith P.T. \& S. Kambhampati 2000. Evolutionary transitions in Aphidiinae (Hymenoptera: Braconidae). Pages 106-113 in A. D. Austin and M. Dowton, eds. Hymenoptera: Evolution, biodiversity and biological control. CSIRO Publishing, Collingwood, Australia.p, 512.

Sobutay U. 2016. Bartın ili Coccinellidae (Insecta: Coleoptera) türleri. Yüksek Lisans Tezi, Bartın Üniversitesi Fen Bilimleri Enstitüsü, Bartın, 111s.

Soydanbay M. 1976. Türkiye'de bitki zararlısı bazı böceklerin doğal düşman listesi. Kısım I, Bitki Koruma Bülteni, 16: 32-46.

Şahbaz A. \& M. Uysal 2006. Konya İli'nde kavaklarda beslenen yaprakbitlerinin (Homoptera: Aphididae) predatör ve parazitoitleri. Selçuk Üniversitesi Ziraat Fakültesi Dergisi, 20 (38): 119-125. 
Tamer A., M. Aydemir \& A. Has 1997. Ankara ve Konya illerinde korunga ve yoncada görülen zararlı ve faydalı böcekler üzerinde faunistik çalışmalar. Bitki Koruma Bülteni, 37(3-4):125-161.

Tezcan S. \& N. Uygun 2003. İzmir ve Manisa yöresi ekolojik kiraz üretim bahçelerinde saptanan Coccinellidae (Coleoptera) türleri üzerinde bir değerlendirme. Türkiye Entomoloji Dergisi, 27 (1): 73-79.

Tiftikci P. 2017. Yozgat ili şeker pancarı üretim alanlarında saptanan Coccinellidae (Coleoptera) türleri üzerinde araştırmalar. Erciyes Üniversitesi Fen Bilimleri Enstitüsü Dergisi, 33 (2): 79-90.

Tomanović Z., A. Beyarslan, Ö. Çetin Erdoğan \& V. Žıkıć 2008. New records of Aphid parasitoids (Hymenoptera, Braconidae, Aphidiinae) from Turkey. Periodicum Biologorum, 110: 335-338.

Ulusoy R., G. Vatansever \& N. Uygun 1999. Ulukışla (Niğde) ve Pozantı (Adana) yöresi kiraz ağaçlarında zararlı olan türler, doğal düşmanları ve önemlileri üzerindeki gözlemler. Türkiye Entomoloji Dergisi, 23: 111-120.

Uygun N. 1981. Türkiye Coccinellidae (Coleoptera) Faunası Üzerinde Taksonomik Araştırmalar. Çukurova Ziraat Fak. Yayınları, Adana, $110 \mathrm{s.}$

Uygun N., H. Başpınar, E. Şekeroğlu, S. Kornoşor, A.F. Özgür, İ. Karaca, M.R. Ulusoy \& C. Kazak 1995. GAP Alanında Zirai Mücadele Politikasına Esas Teşkil Edecek Zararlı ve Yararlının Saptanması. GAP Bölgesi Bitki Koruma Sorunları ve Çözüm Önerileri Sempozyumu. 27-29 Nisan 1995, Şanlıurfa, 99-119.

Ülgentürk S., J. Noyes, C. Zeki \& M.B. Kaydan 2004. Natural enemies of coccoidea (Hemiptera) on orchard trees and the neighbouring areas plants in Afyon, Ankara, Burdur Isparta provinces, Turkey, Proceeding of the X International Symposium on Scale Insect Studies, 19-23 April 2004, Adana, Turkey, 361-372.

Vandenberg N.J. 2002. Coccinellidae Latreille, 1807, (Editör: R. H. Arnett \& M.C. Thomas, American Beetles. Vol.2. Polyphaga: Scarabaeoidea through Curculionoidea). Boca Raton, CRC Press, 371-389.

Varlı S.V., G. Vatansever Sakin \& T. Öncül Abacıgil 2013. Edremit Körfezi (Balıkesir/Türkiye) zeytin bahçelerinde farklı tuzak yöntemleri ile toplanan Coccinellidae (Coleoptera) türleri. Türkiye Entomoloji Dergisi, 3 (3): 151- 160.

Yaşar B., M.S. Özgökçe \& İ. Kasap 1999. Van İlinde bulunan coccinellidae (Coleoptera) familyasina bağlı predatör türlerin saptanması üzerinde araştırmalar. Türkiye 4. Biyolojik Mücadele Kongresi, 26-29 Ocak 1999, Adana, Entomoloji Derneği Yayınları, 445-454.

Yerlikaya H., H. Başpınar \& E. M. Yıldırım 2016. Aydın il merkezinde turunç Citrus aurantium L. (Rutaceae) ağaçlarında bulunan Coccoidea üst familyası ile Aphididae ve Aleyrodidae familyaları (Hemiptera)' na bağlı türlerin saptanması, bulaşma oranlarının ve doğal düşmanlarının belirlenmesi. Türkiye Entomoloji Bülteni, 6 (3): 221-230.

Yeşilayer A. 2009. İstanbul ili yeşil alanlarında zararlı akar (Acarina) türlerinin tanımı, yayılışı, önemli türün popülasyon yoğunluğu ve doğal düşmanları üzerinde araştırmalar. Doktora Tezi, Ankara Üniversitesi Fen Bilimleri Enstitüsü, Ankara, 200s.

Yoldaş Z., A. Güncan \& T. Koçlu 2011. Seasonal occurrence of aphids and their natural enemies in Satsuma mandarin orchards in Izmir, Turkey. Türkiye Entomoloji Dergisi, 35 (1): 59-74. 
Kayseri ili süs bitkilerindeki yaprakbitlerinin parazitoitleri ve avc1 Coccinellidleri

Yumruktepe R. \& N. Uygun 1994. Doğu Akdeniz Bölgesi turunçgil bahçelerinde saptanan yaprakbiti (Homoptera: Aphididae) türleri ve doğal düşmanları. Türkiye 3. Biyolojik Mücadele Kongresi Bildirileri, İzmir. 1-12.

Yurtsever S. 2001. A preliminary study on the ladybirds (Coleoptera: Coccinellidae) of Edirne in North-Western Turkey. Turkish Journal of Zoology, 25: 71-75.

Zeren O. \& Düzgüneş Z. 1983. Çukurova Bölgesinde sebzelerde zararlı olan Aphidoidea türlerinin doğal düşmanları üzerinde araştırmalar. Türkiye Bitki Koruma Dergisi, 7 (3): 199-211. 